\title{
Implementation of a capstone course with Formative OSCEs to evaluate preparedness for Advanced Pharmacy Practice Experiences (APPE)
}

\author{
Ashleigh L Barrickman (D), Lena Maynor iD \\ Department of Clinical Pharmacy, West Virginia University School of Pharmacy, USA
}

\section{Keywords}

Advanced Pharmacy Practice Experiences Assessment

Capstone

Objective Structured Clinical Examinations

\section{Correspondence}

Ashleigh L Barrickman

School of Pharmacy

West Virginia University

PO Box 9520

Morgantown

WV 26506

alandis1@hsc.wvu.edu

\begin{abstract}
Background: Capstone courses are well documented in pharmacy programmes, but vary in content, methods, and assessment. Aim: To describe the development and implementation of a capstone course for pharmacy students. Description: Components of the capstone course included clinical reviews, pre-tests, calculations, cases, and formative and summative objective structured clinical examinations (OSCEs). Formative OSCEs were a unique aspect of this capstone course, and were used to help students identify clinical strengths and recognise areas of weakness prior to advanced pharmacy practice experiences (APPE). Evaluation: A total of 72 students completed the capstone course in spring 2018. Student survey data indicated that the format of the course was conducive to learning, particularly the use of formative OSCEs. Conclusion: A capstone course was successfully designed and implemented that assessed a variety of pharmacy knowledge and skills prior to APPE. Student feedback and performance in the course provided insight that led to revisions in the pre-APPE curriculum.
\end{abstract}

\section{Introduction}

Capstone courses have been incorporated into professional pharmacy programmes for many years with goals of preparing students for Advanced Pharmacy Practice Experiences (APPE), providing an opportunity for students to apply knowledge from across the didactic curriculum, improving student confidence, advancing patient care skills, and providing multiple methods for curricular assessment (Conway \& Ahmed, 2012; Beatty et al., 2014; Hirsch \& Parihar, 2014; Lee et al., 2014; Saseen et al., 2017). Such courses allow Pharm.D. programmes to promote critical thinking and problem-solving skills, advance students' ability to appropriately provide patient care, and ensure that students finishing the didactic portion of the Pharm.D. curriculum are ready to proceed to APPE. Additionally, capstone courses help students in the challenging transition from a didactic learner to an independent professional (Hirsch \& Parihar, 2014). Capstone courses that have been described in the literature often incorporate different assessment methods to ensure students are able to integrate knowledge and demonstrate appropriate skills (Hirsch \& Parihar, 2014; Saseen et al., 2017). A combination of formative assessment methods, in which feedback is provided to improve student learning and encourage students to identify strengths and weaknesses, and summative assessment methods, in which student learning is evaluated, are often included in a capstone course (Carnegie Mellon University Eberly Center: Teaching Excellence and Educational Innovation, n.d.).

In professional programmes, factual knowledge is often assessed with traditional methods, including multiplechoice, short answer, and true/false examinations. While these methods effectively determine a student's ability to recall information and identify baseline knowledge, they have limitations for adequately assessing other skills such as communication, problemsolving, critical thinking, and application of knowledge (Hastings et al., 2010; Urteaga et al., 2015). For this reason, traditional assessment methods often require supplementation to assess student competence 
adequately, proficiency in knowledge application, communication abilities and patient care skills (Beatty et al., 2014; Urteaga et al., 2015). An assessment method that can be used to supplement traditional methods is the objective structured clinical examination (OSCE), a performance-based assessment tool that has a well-established role in medical education (Salinitri et al., 2012). Since their implementation in medical education in 1975, OSCEs have become the gold standard for evaluating learner performance in many health professions (Sloan et al., 1995; Shirwaikar, 2015). OSCEs offer an opportunity to connect factual knowledge and clinical application, allowing for the evaluation of communication and interpersonal skills and the ability to resolve complex problems. As such, OSCEs are often ideal assessment methods for capstone courses (Hastings et al., 2010; Austin et al., 2003). The use of OSCEs to evaluate a variety of clinical skills is supported by literature, and OSCEs have been proven to be valid and reliable forms of assessment (Urteaga et al., 2015). The use of OSCEs as a formative teaching tool has been documented in other health professions education programmes and described as a valuable experience for learners (Brazeau, Boyd, \& Crosson, 2002; Farahat et al., 2016). Advantages of formative OSCEs include the ability to assess students with objective criteria while minimising student stress, and the ability to foster learning by allowing students to self-identify areas of weakness and strength (Gupta, Dewan, \& Singh, 2010; Abu Bdair, Abuzaineh, \& Burqan, 2019). Formative OSCEs also provide students with an opportunity to practice for summative OSCEs and gain experience interacting with patients and healthcare providers (Brazeau, Boyd, \& Crosson, 2002). Limitations of formative OSCEs include the possibility for inaccurate assessment data, as students may not adequately prepare due to the lowstakes nature of the exam, and the resource commitment necessary to implement quality OSCEs that are not being used as a summative assessment.

Students in the school of pharmacy programme in West Virginia University School of Pharmacy complete three years of didactic education, followed by a fourth year of experience in APPEs. During an extensive curricular revision, it was determined that a capstone course at the conclusion of the didactic curriculum was needed to ensure APPE-readiness. The purpose of this capstone course was to provide a review of pertinent content as well as assess knowledge and skills needed for successful completion of the upcoming APPE curriculum. Multiple teaching and assessment methods were needed in this capstone course to allow for the attainment of the objectives, which included 1) application of critical thinking and problem-solving skills; 2) communication of medication therapy recommendations to patients and health care providers; 3) identification of medication-related problems; and 4) incorporation of law, ethics, pharmacology and therapeutic knowledge to develop patient care plans. Detailed learning objectives for the capstone course can be found in Table I. Additional assignments were developed to further assess drug information and literature evaluation skills, application of pharmacy-based calculations, and prescription verification skills in both community and hospital settings. This study was approved by the University's Institutional Review Board (IRB).

\section{Description of the course}

This capstone course was a 3-credit hour course implemented in the spring term of the third didactic year of the professional pharmacy curriculum, immediately preceding APPEs. Successful completion of this course is a graduation requirement, and since the course serves as a review of didactic content, students are required to have completed or to be in the process of completing all other didactic courses in the curriculum in order to enrol in the capstone course. Similar to systems-based therapy courses in the pharmacy programme of this university, this capstone course was delivered in a concentrated format, with students meeting for two hours daily over five weeks. To achieve a broad review of content and create multiple types of assessment, the course was divided into three major components, didactic coursework, practicum, and assessment through the Pharmacy Curriculum Outcomes Assessment (PCOA). Practicum is a weekly two-hour session that serves as a hands-on component to complement the didactic sessions for systems-based therapy courses and the capstone course. Didactic course components were completed during regularly scheduled class time, practicum components were completed during regularly scheduled skills development time, and the PCOA was scheduled by school administration prior to the start of the capstone course based on the programme's needs.

As the objective of the capstone course was to evaluate APPE preparedness and ensure students demonstrated at least minimal competence in knowledge and skills from the didactic curriculum, the capstone course was graded on a pass/fail basis. Students were required to achieve at least a $70 \%$ average on all graded components of the capstone course, as well as a grade of "pass" for each pass/fail component in order to achieve a grade of "pass" for the course. As shown in Table II, capstone course components that focused on 
the review of content were graded on a pass/fail basis, and students received a grade of "pass" for completion of pass/fail assignments regardless of performance. With the exception of the summative OSCE, components of the capstone course that focused on the assessment of APPE readiness were letter-graded. By design, OSCEs are used to demonstrate minimal competence in a specific set of knowledge and skills and are recommended to be graded on a pass/fail basis
(Austin et al., 2003; Sturpe, 2010). Formative OSCEs and the summative OSCE were graded on a pass/fail basis in this capstone course. Students achieved a score of "pass" on formative OSCEs for completing the OSCE, as the main purpose was to provide feedback to the students. In order for students to achieve a score of "pass" on the summative OSCE, students were required to achieve a score at or above the predetermined cut score.

Table I: Learning objectives for the capstone course

(1) Display professional demeanour and behaviour.

- Demonstrate respect for everyone encountered during class activities, including instructors, classmates, and standardized patients

- Accept responsibility and accountability for class-related responsibilities

(2) Articulate appropriate time management techniques.

- Prioritise tasks and responsibilities for completion in a clinical setting

- Describe commune responsibilities, including time management skills that may be included as part of an APPE rotation

(3) Display self-awareness and self-improvement.

- Identify and reflect on personal strengths and weaknesses

- Demonstrate self-directed pursuit of new knowledge and skills

(4) Demonstrate patient-centred practice skills.

- Display patient and caregiver compassion and empathy

- Consider patient and caregiver needs and desires regarding care

- Represent the patient's best interest with respect to providing pharmacy care

(5) Demonstrate effective verbal and non-verbal communication skills.

- Communicate in a manner appropriate for the intended audience

- Use proper grammar and vocabulary

- Maintain appropriate eye contact and body posture

- Minimise distracting behaviours

- Speak clearly

(6) Demonstrate effective written communication skills.

- Clearly articulate message through a written medium

- Communicate in a manner appropriate for the intended audience

(7) Demonstrate cultural competence.

- Appropriately interact with persons for backgrounds or cultures different from one's own

- Acknowledge the attitudes, opinions and needs of persons from different backgrounds or cultures

(8) Articulate approaches and methods for interacting as part of an interprofessional team.

- Explain the pharmacist's role in interprofessional interactions in common pharmacy practice settings

- Develop plans for preventing and resolving communication breakdown among members of interprofessional teams

(9) Demonstrate knowledge of common disease states, including pharmacy-specific knowledge needed to complete APPE successfully.

- Identify and explain common indications, dosing, monitoring parameters, and safety considerations of relevant medications

- Identify medication-related problems, such as drug interactions, gaps in treatments and monitoring

- Describe the pathophysiology of common disease states

- Articulate pharmacy-related standards of care for common disease states

- Apply basic science principles, including pharmacokinetics and biopharmaceutics, to address medication-related problems

(10) Demonstrate the pharmacy skills needed to successfully complete APPE

- Apply the Pharmacists' Patient Care Process to case-based simulation, standardized patients and case exams

- Correctly perform pharmacy-related calculations

- Demonstrate appropriate methods and procedures for dispensing medication in community and hospital settings

- Identify errors and omissions in prescriptions or inpatient medication orders

- Demonstrates appropriate technique for compounding medications

- Accurately measure blood pressure in a simulated environment

- Accurately use point of care testing equipment to measure blood glucose, A1c, cholesterol and INR

- Explain pertinent counselling points for insulin and inhaler administration

- Accurately perform IV admixing

(11) Apply problem-solving techniques

- Assess components of simple and complex problems

- Identify multiple potential solutions

- Assess chosen course of action

(12) Apply critical thinking techniques - Formulate clarifying questions 
- Seek information from credible sources

- Use reasoning to assess problems

(13) Identify relevant background information to clarify drug information questions

- Clearly articulate actual information need

(14) Develop applicable, concise responses to drug information questions

- Appropriately communicate relevant information without extraneous or unclear information

(15) Effectively interpret primary literature

- Identify important factors that impact study adequacy, including design and methods

- Determine key strengths and weaknesses of a study

- Identify and assess the use of common statistical analyses

- Articulate the overall impact of a clinical study

(16) Identify potential quality and medication safety issues

- Explain methods to resolve prescription or inpatient medication order errors

(17) Identify national standards, guidelines and best practices related to pharmacy

- Describe methods for ensuring continuity of care for patients transitioning between settings

- Demonstrate appropriate technique in administering immunizations, epi-pens, insulin and inhalers

APPE: Advanced pharmacy practice experiences; INR: International normalized ratio

Table II: Evaluation of learning and grading for the capstone course

\begin{tabular}{|c|c|c|}
\hline Evaluation method & $\begin{array}{l}\text { Associated ability-based } \\
\text { outcomes (see Table I) }\end{array}$ & Per cent of grade \\
\hline Pharmacy curriculum outcomes assessment (PCOA) & 9,12 & 15 \\
\hline Calculations pretest or post-test & 10,12 & 15 \\
\hline Literature review practicum & $6,12,13,14,15,16$ & 10 \\
\hline Mini-case quizzes (4 quizzes) & $9,10,11,17$ & 40 (10\% per quiz) \\
\hline $\begin{array}{l}\text { Community errors and omissions } \\
\text { (Non-interactive station from teaching OSCE 1) }\end{array}$ & 10,12 & 5 \\
\hline $\begin{array}{l}\text { Written SOAP note } \\
\text { (Non-interactive station from teaching OSCE 2) }\end{array}$ & $6,9,10,11,12$ & 5 \\
\hline $\begin{array}{l}\text { Hospital errors and omissions assignment/Opioid conversion } \\
\text { (Non-Interactive station from teaching OSCE 3) }\end{array}$ & 10,12 & 5 \\
\hline $\begin{array}{l}\text { Hospital calculations assignment } \\
\text { (Non-Interactive station teaching OSCE 4) }\end{array}$ & 10,12 & 5 \\
\hline Formative OSCEs 1-4 & $1,4,5,6,7,8,9,10,11,12,17$ & Pass/Fail* \\
\hline Systems review pretests ( 8 ) & 9,11 & Pass/Fail \\
\hline Mini-cases (8) & 2,12 & Pass/Fail \\
\hline Portfolio class session & $2,3,12$ & Pass/Fail \\
\hline Summative OSCE & $1,4,5,6,7,8,9,10,11,12,17$ & Pass/Fail \\
\hline
\end{tabular}

PCOA: Pharmacy Curriculum Outcomes Assessment; OSCE: Objective structured clinical examination

*Students were not required to achieve the cut score for the formative OSCEs - students received a "pass" for the OSCE for participation

The sequencing of the capstone course components followed the order of the systems-based therapy course series. Discussions, assignments, and assessments were linked to the topics discussed each week. The components of the course are described in more detail below, and the course schedule is depicted in Figure 1 . Students spent approximately 12 contact hours each week as part of this capstone course and additional time outside of class completing pretests and preparing for course assessments.

\section{Pharmacy curriculum outcomes assessment (PCOA) exam}

The PCOA is a validated tool developed by the National Association of Boards of Pharmacy (NABP) required for all students to take prior to beginning APPEs according to the 2016 Standards from the Accreditation Council for Pharmacy Education (ACPE). The PCOA is used in many colleges of pharmacy for curricular assessment and individual assessment. One study indicated that approximately $83 \%$ of colleges of pharmacy administer the PCOA to third-year students, and approximately $20 \%$ of schools use the PCOA in a medium to high stakes manner (Gortney et al., 2019). All students at our institution are required to complete the PCOA during the spring term of the third didactic year of the pharmacy curriculum. As one of the main goals of this capstone course was to assess student knowledge prior to APPE, the PCOA was incorporated into the capstone course to encourage students to put forth their best effort on the examination. Each student's score on the PCOA was incorporated into the final grade of the capstone course. The student's scaled score and the National Year 3 Normed Reference Total Scaled Score (NNRS) were used to determine the student's grade for the capstone course based on a predetermined scale, shown in Table III. 


\begin{tabular}{|c|c|c|c|c|c|c|}
\hline \multicolumn{7}{|c|}{ Capstone Course Schedule } \\
\hline Week: & Monday & Tuesday & Wednesday & Thursday & Thursday (Practicum Session) & Friday \\
\hline Week 1 & & & & Orientation & $\begin{array}{l}\text { Formative OSCE } 1 \text { (Self-care and } \\
\text { vaccination cases) } \\
\text { Non-Interactive station: Community } \\
\text { Errors and Omissions } \\
\text { Formative OSCE } 1 \text { Debrief }\end{array}$ & $\begin{array}{l}\text { Systems Review } \\
\text { (Rheumatology and Pain) }\end{array}$ \\
\hline Week 2 & $\begin{array}{l}\text { Systems Review } \\
\text { (Pulmonology) }\end{array}$ & $\begin{array}{l}\text { Mini-Cases } 1 \text { (Rheumatology } \\
\text { and Pulmonology) }\end{array}$ & Calculations Pre-Test & $\begin{array}{l}\text { Systems Review } \\
\text { (Cardiology) }\end{array}$ & $\begin{array}{l}\text { Formative OSCE } 2 \text { (Rheumatology } \\
\text { and Pulmonology cases) } \\
\text { Non-Interactive Station: SOAP Note }\end{array}$ & Calculations Review \\
\hline Week 3 & Formative OSCE 2 Debrief & $\begin{array}{l}\text { Systems Review } \\
\text { (Endocrinology) }\end{array}$ & $\begin{array}{l}\text { Mini-Cases } 2 \\
\text { (Endocrinology) and Drug } \\
\text { Information Assignment (Cardiology) }\end{array}$ & Portfolio Discussion & $\begin{array}{l}\text { Formative OSCE } 3 \text { (Cardiology and } \\
\text { Endocrinology cases) } \\
\text { Non-Interactive Station: Hospital } \\
\text { Errors and Omissions/Opioid } \\
\text { Conversion }\end{array}$ & $\begin{array}{l}\text { Systems Review } \\
\text { (Gastroenterology) }\end{array}$ \\
\hline Week 4 & Formative OSCE 3 Debrief & $\begin{array}{l}\text { Systems Review } \\
\text { (Infectious Diseases) }\end{array}$ & $\begin{array}{l}\text { Mini-Cases } 3 \\
\text { (Gastroenterology and Infectious } \\
\text { Diseases) }\end{array}$ & APPE Orientation & $\begin{array}{l}\text { Formative OSCE } 4 \text { (Gastroenterology } \\
\text { and Infectious Diseases cases) } \\
\text { Non-Interactive Station: Hospital } \\
\text { Calculations }\end{array}$ & $\begin{array}{l}\text { Systems Review } \\
\text { (Nephrology) }\end{array}$ \\
\hline Week 5 & Formative OSCE 4 Debrief & $\begin{array}{l}\text { Systems Review } \\
\text { (Neurology/ Psychiatry) }\end{array}$ & $\begin{array}{l}\text { Mini-Cases } 4 \text { (Nephrology and } \\
\text { Neurology/ Psychiatry) }\end{array}$ & Calculations Post-Test & Literature Evaluation Assignment & APPE Orientation \\
\hline
\end{tabular}

APPE: Advanced Pharmacy Practice Experiences; OSCE: objective structured clinical examination

Note: Students completed the Pharmacy Curriculum Outcomes Assessment (PCOA) prior to the start of this course.

Figure 1: Schedule for a comprehensive assessment of practice course

Table III: Capstone course grade conversion based on student scaled scores on PCOA

\begin{tabular}{cc}
$\begin{array}{c}\text { Student scaled score on } \\
\text { PCOA exam }\end{array}$ & $\begin{array}{c}\text { The corresponding grade for } \\
\text { the capstone course }\end{array}$ \\
\hline$\geq 423$ & $100 \%$ \\
$405-422$ & $95 \%$ \\
$387-404$ & $90 \%$ \\
$370-386$ & $85 \%$ \\
$352-369$ & $80 \%$ \\
$335-351$ & $75 \%$ \\
$317-334$ & $70 \%$ \\
$300-316$ & $65 \%$ \\
$282-299$ & $60 \%$ \\
$264-281$ & $55 \%$ \\
$247-263$ & $50 \%$ \\
$230-246$ & $45 \%$ \\
$213-229$ & $40 \%$ \\
$196-212$ & $35 \%$ \\
$179-195$ & $30 \%$ \\
$162-178$ & $25 \%$ \\
$145-161$ & $20 \%$ \\
$128-144$ & $15 \%$ \\
$111-127$ & $10 \%$ \\
$94-110$ & $5 \%$ \\
$\leq 93$ & $0 \%$ \\
&
\end{tabular}

\section{Didactic components of the capstone course}

Therapeutic systems reviews

Students were required to attend eight, two-hour review sessions that corresponded to each systemsbased therapy course in the curriculum through the autumn term of the third didactic year of the pharmacy curriculum. These systems review presentations typically consisted of a brief overview of important concepts, clinical pearls, and application cases focused on medication management and therapeutic recommendations. These sessions served as a reinforcement of previous content. New content was not included in these sessions, with the exception of updates to relevant clinical practice guidelines. The systems review sessions were developed and facilitated by the coordinator of the corresponding systems-based therapy course or a content expert at the institution. An overview of the specific topics that were included in each systems review is depicted in Figure 2. Topics were chosen based on disease state prevalence and classification as a Tier 1 topic as determined by the American College of Clinical Pharmacy (ACCP) 2016 Pharmacotherapy Didactic Curriculum Toolkit (Schwinghammer et al., 2016).

Prior to each systems review, students were required to complete an online pretest on the associated content. Pretests included multiple question formats, including multiple-choice, fill-in-the-blank, select-allthat-apply, and true/false. Course coordinators compiled pretest questions from standard NAPLEX preparation materials (Bonsell, 2018; Brooks et al., 2018). Online pretests were designed to allow students to assess their baseline understanding of content prior to the review session and to prepare students for the format of NAPLEX questions. Students were permitted to complete the pretest multiple times, and students were able to view the rationale for the answer upon submission for all questions that had been answered correctly. 


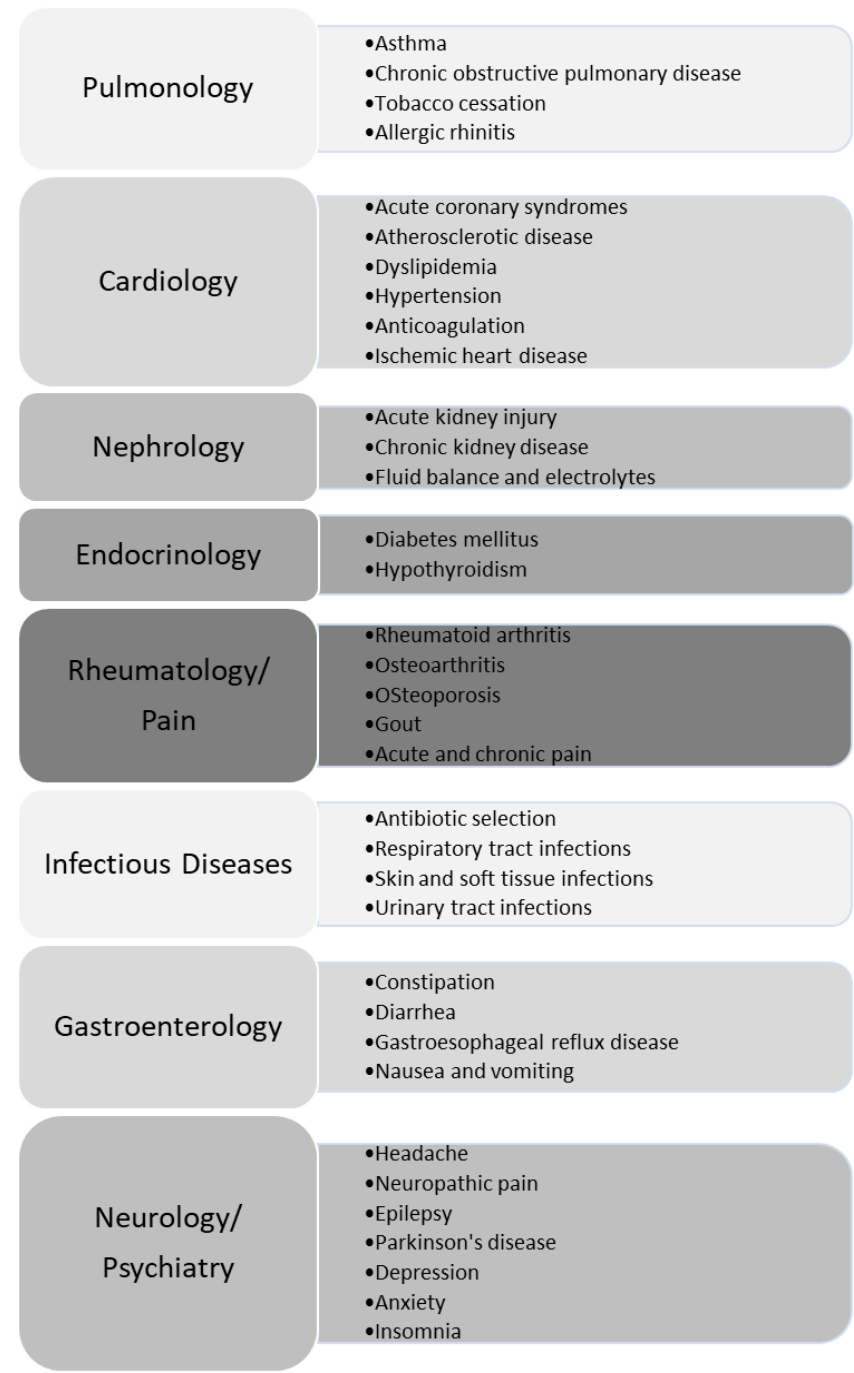

Figure 2: Overview of topics included in therapeutic systems reviews

\section{Mini-cases and quizzes}

Students individually completed patient cases that included the clinical application of content. Each minicase class session included two short case vignettes with four to five questions each, which required students to develop care plans and provide patient education. The specific diseases addressed in the minicases were intentionally selected to highlight a common disease within the corresponding systems reviewed that week of class and to differ from the diseases or specific problems within the formative OSCE for that week. Within the curriculum, this type of case vignette is referred to as a mini-case to distinguish the format from the more in-depth patient cases included within the practicum portion of our systemsbased therapy courses. Eight mini-cases (four class sessions) were completed in the course. Students were given approximately one hour to complete two minicases, after which an instructor provided a one-hour debrief (approximately 30 minutes per mini-case) on important concepts and clinical pearls to help reinforce important and complex content.

Following the mini-cases and debrief, students completed a quiz with 20-multiple choice questions related to the concepts discussed in the debriefing session to assess student knowledge and understanding of the material. The mini-cases and quizzes were developed by course coordinators based on topics that were covered in the corresponding systems reviews.

\section{Calculations}

All students were required to complete a pharmacy calculations pretest during the first week of the course. Calculation exam questions were developed by the faculty member who teaches the calculations course in the first professional year of the curriculum, and all calculation exams were graded by that faculty member to maintain consistency in grading. Students who scored $<80 \%$ on the pretest were required to attend a two-hour calculations review session and to take a calculations post-test. Any student who scored $\geq 80 \%$ on the pretest was excused from attending the calculations review session and completing the calculations post-test but were permitted to attend the calculations review session or complete the post-test if desired. Practice problems were provided to students prior to the post-test.

\section{Drug information assignment}

Students were asked to develop a response to a drug information question, and student responses were evaluated based on 1) completeness and accuracy; 2) content citation; 3) resources used; 4) writing quality; 5) reference format. Students had one hour of a twohour class session to complete the assignment. The focus of the drug information question corresponded to the class content for that week, and all students answered the same drug information question. The faculty member who teaches drug information content in the curriculum developed the drug information question and graded all assignments to maintain consistency.

\section{Miscellaneous didactic course components}

In addition to the main course components described above, students were also required to attend a class session on portfolios, which are used throughout the curriculum for students to self-reflect and develop 
plans for performance-based improvement. An overview of portfolio requirements during APPE was provided during the two-hour class session. Students were also required to attend two APPE Orientation sessions in this course, which included information pertinent for preparing for APPE.

\section{Practicum components of the capstone course}

Literature review assignment

Students were required to complete a literature review assignment in which they applied concepts regarding analysis and critique of a journal article to answer questions about a selected study. Students were provided with the study approximately one week prior to the practicum. Students were given two hours to complete a 56-question assignment related to the study, which consisted of multiple-choice, select-allthat-apply, and short answer questions. All students completed the assignment on the same study. The faculty who teaches medical literature evaluation and literature review developed the questions and graded all assignments to maintain consistency.

\section{Formative Objective Structured Clinical Examinations (OSCES)}

This capstone course placed significant emphasis on the use of formative OSCEs as a learning tool to allow students to apply clinical concepts to real-life scenarios. Students completed four formative OSCEs, scheduled weekly during four practicum sessions of the course. OSCEs were completed in the simulation centre available to all programmes within the health sciences centre. For the OSCEs, the simulation centre's examination rooms are primarily used, which are designed to resemble a patient exam room in an ambulatory care clinic. Each examination room contains a desktop computer to allow students to access an electronic health record (EHR) and drug information references. From the proctor station in the simulation centre, course coordinators were able to view the computer screen of all rooms to ensure the appropriate use of resources. Faculty evaluators used the computer laboratory in the simulation centre to evaluate the OSCEs in real-time, allowing for a quick turn-around of scoring so performance could be discussed during the OSCE debrief.

Each formative OSCE contained 2-3 interactive stations that required students to interact with a standardized patient in a scenario that tested the student's clinical knowledge and communication skills, as well as one or more non-interactive stations. The formative OSCE blueprint was structured such that OSCE stations corresponded to the systems reviews and mini-cases that had most recently occurred in the capstone course. Case writers for OSCE stations were asked to develop cases with specific medical conditions or patient complications, so that common diseases could be reinforced without being repetitive with mini-case content. Course coordinators also structured the OSCE blueprint to ensure that cases for each week required students to resolve medication-related problems in a variety of settings, including community pharmacies, inpatient settings and ambulatory care clinics.

Students were given eight minutes to complete each interactive formative OSCE station. Each week, one to two of the interactive OSCE stations were evaluated in real-time by either a faculty member, adjunct pharmacist preceptor, pharmacy resident, or fourthyear pharmacy (P4) student. Students completing the course were randomly assigned a peer review partner and were responsible for evaluating their classmate's performance on one OSCE station each week. Stations were evaluated using an analytical checklist, with clinical components specific to each case and a global assessment rubric to assess communication skills. The analytical checklists included $12-20$ items specific to each case in the areas of gathering information, management strategies and monitoring/follow-up. Feedback was provided to students during a debriefing session that followed each OSCE. Students had access to their analytical checklist and global assessment scores for each station and were provided with the cut score for each OSCE station and the overall OSCE exam cut score during the debrief. Cut scores were determined for each OSCE station by a team of three pharmacists, using a modified Angoff method. Students received credit for participation in formative OSCEs. Although students were not required to achieve the cut score to pass formative OSCEs, cut scores for each station and overall weekly formative OSCE cut scores were provided to students during the weekly debrief. Students could compare their OSCE score to the cut score to determine if their performance would have resulted in either a pass or fail had the OSCE been summative.

While the interactive stations of the formative OSCES were pass/fail to provide formative feedback to the students and allow students to identify areas of strength and areas for improvement, non-interactive stations included skills that the course coordinators felt students needed to demonstrate competency in prior to starting APPE. Non-interactive stations included community and hospital prescription verification exercises, development of a SOAP note, hospital calculations, and pharmacokinetic dosing vignettes. The specific skills/knowledge that was assessed on each 
non-interactive station was announced to students prior to the OSCE. These stations were developed by course coordinators and letter-graded, and students were provided with feedback on their performance from course coordinators during the OSCE debrief, as well as individualised written feedback. A single course coordinator graded the non-interactive station each week to maintain consistency in grading.

\section{Final examination}

The final examination in this course was a summative OSCE with eight interactive stations. Students were required to achieve equal to or above the predetermined OSCE cut score in order to obtain a passing grade on the summative OSCE, and consequently, in the course. The format for the final summative OSCE was very similar to that of the formative OSCEs, with the exception that students were allowed ten minutes for each interactive OSCE station. Similar to formative OSCEs, course coordinators created a blueprint for the final OSCE to ensure that there was a mixture of simple and complex cases and disease states. No P4 student evaluators were used for the summative OSCE, and non-interactive stations were not included. Due to resource commitment, including time, standardized patients and evaluators, it was not possible to complete all eight stations in one day, so students completed the summative OSCE over two days by completing four stations per day. Additional security measures were taken to maintain the integrity of the summative OSCE stations due to the high stakes nature of the exam. Students were required to check-in prior to the start of the OSCE and turn in all electronic devices capable of messaging, including phones, smartwatches, and laptops. As each student group completed the OSCE, the group was escorted to the pharmacy skills laboratory by a teaching assistant and sequestered without access to electronic devices until the final group for the day formally checked in to begin their OSCE. During sequestration, students were monitored by a teaching assistant and provided with movies, games, snacks and lunch. Students were not provided feedback on the final OSCE, other than their final composite score for all eight stations. Due to the high stakes nature of the summative OSCE, a review process was developed in which two faculty reviewers (in addition to the initial station evaluators) independently watched and scored recordings of stations for any student with a failing grade on the overall summative OSCE.

At the conclusion of this capstone course, students were provided with an anonymous, voluntary course survey to provide feedback on the different components of the course. The survey included Likert-scale and open-ended questions about the main course components, including systems reviews, mini-cases, pretests, calculations, OSCEs, and the overall course.

\section{Evaluation}

Seventy-two students completed the capstone course in spring 2018. All students achieved a grade of pass for the course.

\section{Graded course components}

Average student scores for each of the graded components of the capstone course are depicted in Table IV. Of the graded components of the course, the average percentage grades were lowest on the drug information assignment, $55.7 \%(S D=8.8)$ and the medical literature evaluation assignment, $76.5 \% \quad(S D=9.0)$. Average percentage grades were highest on mini-case quizzes, with the lowest mini-case quiz average score of $88.3 \%(S D=7.7)$ and the highest mini-case quiz average score of $94.7 \%(S D=4.2)$. The average score assigned to PCOA performance in the course was $84.8 \%(S D=4.4)$, which corresponded to an average scaled PCOA score of 352 (SD = 39.9). Performance on the PCOA was not correlated with either formative OSCE or summative OSCE performance. Weak correlations, with trends toward significance, between PCOA and average performance on the letter-graded portion of the capstone course and overall grade point average (GPA) at the conclusion of the pre-APPE curriculum, were found ( $r=0.23, p=0.06$ and $r=0.22, p=0.06$, respectively).

Table IV: Graded components of the capstone course

\begin{tabular}{lcc}
\hline Capstone course component & $\begin{array}{c}\text { Average } \\
\text { grade (\%) }\end{array}$ & $\begin{array}{c}\text { Standard } \\
\text { deviation }\end{array}$ \\
\hline PCOA* & 84.8 & 4.4 \\
Mini-Case Quiz 1 (Pulmonology & 94.7 & 4.2 \\
and Rheumatology) & 91.4 & 11.7 \\
Mini-Case Quiz 2 (Endocrinology) & 88.3 & 7.7 \\
Mini-Case Quiz 3 (Gastrointestinal & & \\
and Infectious Diseases) & 90.4 & 8.0 \\
Mini-Case Quiz 4 (Nephrology and & & \\
Neurology/Psychiatry) & 53.7 & 13.4 \\
Calculations pretest & 89.1 & 7.8 \\
Calculations post-test & 55.7 & 8.8 \\
Drug information question & 76.5 & 9.0 \\
Literature review assignment & 71.1 & 16.3 \\
Community errors and omissions & 85.7 & 13.1 \\
Written SOAP note & 82.5 & 14.5 \\
Hospital errors and omissions & 80.4 & 15.3 \\
Hospital calculations & \\
\hline PCOA: Pharmacy curriculum outcomes assessment \\
*This was the score assigned to PCOA performance for the capstone \\
course grade book and corresponds to an average scaled PCOA \\
score of 352.
\end{tabular}




\section{Pass/Fail course components}

Students "passed" formative OSCEs by completing each station; however, as part of their formative feedback, students were given their score to compare against the predetermined cut score. The average "pass" rate (i.e., achievement equal to or above the predetermined cut score) for individual interactive formative OSCE stations was $79.2 \%(S D=12.2)$, and the average overall "pass" rate for formative OSCEs was $84.7 \%$ (SD=13.9). In general, students performed best on global assessment measures, with an unweighted global assessment average across all stations of $80.7 \%$ $(S D=17.2)$. The unweighted averages for analytical checklist items were gathering appropriate information, $\quad 71.2 \% \quad(S D=21.6), \quad$ therapeutic management strategies, $55.4 \% \quad(S D=28.8)$, and monitoring and follow-up, $46.5 \%(S D=37.2)$. For the summative OSCEs, based upon the calculated cut scores for each station, the average pass rate for individual stations was $87.75 \%$ (SD=9.2). All students passed the overall summative OSCE. Of note, the summative OSCE multi-reviewer process described above was implemented for four students, and it was determined that the overall OSCE score was a pass for each of the four students. Additional information regarding the scoring of pass/fail components of the capstone course is shown in Table V.

Table V: Pass/fail components of the capstone course

\begin{tabular}{lcc}
\hline \multicolumn{1}{c}{$\begin{array}{c}\text { Capstone course } \\
\text { component }\end{array}$} & $\begin{array}{c}\text { Average score } \\
\text { (\%) }\end{array}$ & $\begin{array}{c}\text { Standard } \\
\text { deviation }\end{array}$ \\
\hline Rheumatology Pretest & 72.0 & 17.0 \\
Pulmonology Pretest & 61.2 & 17.6 \\
Cardiology Pretest & 64.2 & 21.5 \\
Endocrinology Pretest & 73.2 & 18.2 \\
Gastrointestinal Pretest & 67.6 & 6.7 \\
Infectious Diseases Pretest & 54.6 & 8.1 \\
Nephrology Pretest & 57.6 & 7.1 \\
Neurology/Psychiatry & 53.0 & 17.6 \\
Pretest & & \\
Teaching OSCE 1 & 72 & 9.8 \\
Teaching OSCE 2 & 73 & 7.9 \\
Teaching OSCE 3 & 65 & 9.3 \\
Teaching OSCE 4 & 72 & 6.9 \\
Summative OSCE & 76 & 7.4 \\
\hline
\end{tabular}

OSCE: Objective structured clinical examination

Prior to the summative OSCE, 58 students (80.5\%) completed the course survey, which is summarised in Table VI. A majority of students indicated the overall quality of the course and their learning within the course was either good or excellent $(94.8 \%$ for both survey items).
Table VI: Student survey responses for the capstone course

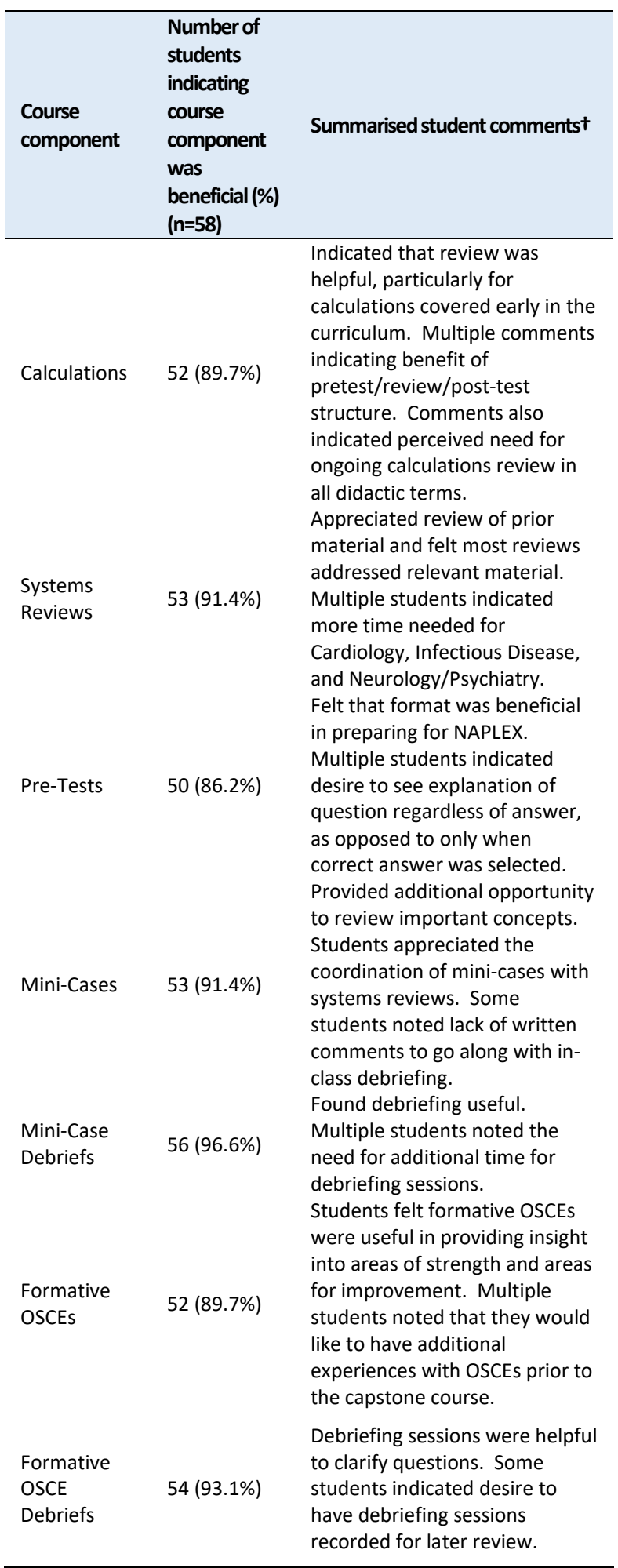

NAPLEX: North American Pharmacists Licensure Exam; OSCE: objective clinical structured examination ${ }^{\dagger}$ Comments were reviewed by a course coordinator, and themes that were mentioned in comments by multiple students were summarised 
Student comments were positive overall, with many comments noting the value of a comprehensive review of material from systems-based therapy courses and their agreement that the course is best suited to a pass/fail format. Multiple students indicated enjoyment and benefit from the emphasis on interactive components in the course. A majority of students (93.1\%) agreed or strongly agreed that the course was well-organised. However, several student comments indicated displeasure that the summative OSCE would have to be passed in order to pass the course.

Many student comments focused on material that they felt needed additional reinforcement throughout each term prior to the capstone, including basic pharmacy calculations, pharmacokinetics, brand/generic names of Top 300 medications, and dosing of Top 300 medications. A number of students indicated that a formative OSCE at the end of each term of the systemsbased therapy course series would be valuable.

\section{Future implementation}

Student performance across course activities, as well as the student course evaluation, have led to some planned changes within the course. The primary limitation identified by student course evaluations related to therapeutic systems reviews was the lack of time to adequately review several systems. Moving forward, reviews for cardiology, neurology/psychiatry, and infectious disease will be allotted four hours (two class sessions). Systems reviews are assigned to a content expert, typically the coordinator of the corresponding systems-based therapy course within the curriculum. The incorporation of a large number of instructors into the course creates some variation in the expectation of students during the session, as some faculty provide a traditional lecture while others provide the review as an active learning session. Student feedback indicates the desire for consistency across reviews. In future iterations of the course, the coordinators will work with instructors to facilitate a more consistent experience for students. Additionally, the range of performance on online pretests for therapeutic systems reviews suggests the possibility that some students consistently used the pretests as intended, while others did not. While the course coordinators have determined that the pretests are most appropriate as a pass/fail assignment, some incentive to perform well on the pretest will be incorporated in future iterations of the course, such as allowing top tier performers the opportunity to select their time assignment for the summative OSCE.
Grading mini-cases on a pass/fail basis, with a lettergraded quiz, mirrors the use of this format in other courses within our curriculum. However, a limitation of this format includes the lack of individualised student feedback, which is challenging to overcome as multiple graders would be necessary to return graded assignments back to students in a timely fashion. Additionally, the pass/fail nature of the assignment creates the possibility that students may not take the completion of the mini-case seriously. Furthermore, since the cases, debrief, and quiz were part of the same class session, some sessions felt rushed, which was noted in student comments. Because the course coordinators are not able to address the lack of needed graders for the foreseeable future, efforts will be made in future offerings of the course to increase the level of knowledge and application skills required to successfully complete the accompanying mini-case quiz. The accompanying assessment will be moved to subsequent class sessions, which will allow additional time for in-person case debriefing and discussion. The assessment will be restructured to include additional questions from corresponding therapeutic systems reviews, mini-case sessions, and associated with top 300 drugs.

While generally well-received, some student course evaluations indicated a need for a review of calculation materials prior to the calculations pretest early in the course. Because of the need to devote in-class time to systems reviews, the coordinators do not plan to hold a pre-exam calculations review in the future. To address this issue, the coordinators plan to release a self-study calculations review for students that would like to complete practice questions prior to the pretest.

Overall performance on the drug information and medical literature assignments indicated a need for additional review and reinforcement of the skills necessary to complete the assignments. In the initial course design, the inclusion of drug information and medical literature review was not included, as reinforcement of relevant content and skill is embedded across multiple courses in the curriculum. The course will remain limited to the current number of credit hours, which along with the identification of other material that needs to be expanded, makes addressing this gap a challenging challenge. Self-study resources, including a recorded drug information review, biostatistics review, and a biostatistics pretest, will be included in future course offerings, and the impact of these interventions will be assessed.

While OSCEs were very well received among students, some limitations have been identified. While the use of an analytical checklist and rubric for global 
assessment improves inter-rater variability, the course coordinators have identified the need for additional OSCE evaluation training to address variations in evaluator expectation and scoring. Additionally, feedback from students and evaluators indicated some differences in the information provided by standardised patients. The course coordinators for the capstone course have developed an online OSCE evaluator training which will be required for future evaluators, with the intent of clarifying expectations of student performance on the global assessment and analytical checklist and decreasing interrater variability. Students may also be required to complete the evaluator training in order to improve the peer evaluation process and increase consistency with peer feedback. Finally, the course coordinators have expanded the time allotted prior to the start of each OSCE to clarify standardised patient questions regarding their assigned case, as well as an expanded standardised patient training prior to the final OSCE.

Several additional opportunities for improvement in this course exist, including the incorporation of nonclinical content into the course, a more rigorous assessment of broad knowledge aside from the PCOA, and the potential for providing additional individualised study resources in specific content areas for students that do not achieve the station cut scores within a formative OSCE. While the concentrated format for course delivery and sequencing following the completion of systems-based therapy courses allows students adequate time to devote to the course and ensure that all systems-based therapy content has been completed prior to the start of the capstone course, the format also limits the ability for the course to be revised to increase the credit and contact hours without adversely affecting the systems-based therapy courses delivered in the same term. Finding a balance between broad content review, appropriate assessments, and timely feedback will continue to be a challenge, given the course structure. Some small accommodations are planned in order to make adequate class sessions available for needed revisions to the course. These accommodations include adding two extra calendar days for the course and moving the calculations exams and the medical literature assignment into the exam block time allotted to courses in the third year of the programme, which is outside of normal class time.

The incorporation of the PCOA into the course provides needed broad knowledge assessment and creates an incentive for students to take the assessment seriously. A significant limitation of the use of the PCOA in the course is the timing of the assessment of third-year students in our curriculum. The PCOA date is determined by the school administration and is based on the needs of the programme. The PCOA is typically scheduled early in the spring term, prior to the start of the capstone course. Student feedback indicated that students who perform poorly on the assessment feel they are at risk for failing the course before the rest of the course begins. It is unlikely that the timing of PCOA administration will change in future years; however, course coordinators are considering options to mitigate the anxiety created by releasing PCOA scores, including releasing PCOA scores after some other graded elements of the course have been completed and offering to meet with individual students to discuss their concerns.

In addition to course changes, changes to the institutional curriculum have been implemented as a result of student performance and feedback from this course. Plans to incorporate prescription verification activities and formative OSCEs into didactic courses prior to the capstone course have been made. A detailed report of formative and summative OSCE station performance was developed, highlighting specific skills and content for which the overall class scored well or scored poorly. This information was presented to the faculty at the institution so that individual coordinators could make adjustments in their courses as they deemed appropriate. Further, student-level data from each assessment was provided to the Director of Assessment for the PharmD programme. This data will allow the Director of Assessment, in conjunction with the Educational Outcomes Assessment Committee, to evaluate trends in performance across subject matter and skills, as well as make recommendations for individual courses in the curriculum regarding potential areas for additional education, practice, or assessment.

Multiple PharmD programmes have published descriptions of capstone courses (Conway \& Ahmed, 2012; Beatty et al., 2014; Hirsch \& Parihar, 2014; Lee et al., 2014; Hobson, Johnston, \& Spinelli, 2015; Saseen et al., 2017). There appear to be common elements across several of these courses, such as the inclusion of patient cases and OSCEs. However, there is considerable variability in capstone course design, such as varying credit hours and the inclusion of nontherapeutics material. Even within the common elements of patient cases and OSCEs, there is variation in the depth and complexity of patient cases and the overall size of the OSCEs. The capstone course implemented at this institution emphasised formative OSCEs as a learning method, which required extensive planning during the year prior to the first offering of the course. Two day-long OSCE writing workshops were held in the summer prior to the first spring term 
offering, in which faculty and other local pharmacists were asked to develop, validate, and set the passing scores for OSCE stations. After the workshops, the course coordinators reviewed all stations to determine the need for minor changes, prop development, and recruitment of standardised patients. The simulation centre at this institution alleviated a significant burden related to identifying and scheduling appropriate standardised patients. Additionally, with the use of the simulation centre's learning management system, interactive OSCE stations could be recorded, station evaluations could be completed electronically, and reports were easily generated based on the evaluation data.

Another common theme in descriptions of capstone courses is the faculty intensive nature of the course design. This capstone course is co-coordinated by two faculty members in the clinical department. The coordinators share responsibilities for preparing online pretests, developing mini-cases, leading mini-case debriefs, preparing mini-case quizzes, developing the blueprint for formative and summative OSCEs, leading OSCE writing workshops to develop new cases, scheduling OSCE space and resources with the health sciences simulation centre, developing OSCE props, facilitating the logistics of the OSCEs, and leading formative OSCE debriefing sessions. Additional faculty presented therapeutic systems reviews, prepared the calculations examinations, developed and graded the drug information and literature evaluation assignments, and served as OSCE evaluators. Even with the extensive faculty assistance in the class, additional faculty time assigned to the course would be necessary to address some previously identified limitations, such as the lack of graded mini-cases.

In addition to being faculty intensive, there was a significant cost required to implement the course relative to other courses in the curriculum. The highest cost of this course was related to formative and summative OSCEs. Most of the cost of using the simulation centre for the OSCEs was included as part of the school of pharmacy's standard fee to the simulation centre, which is used for recruitment and training of standardised patients, use of simulation equipment and simulation centre staff time. The OSCE writing workshops were delivered as accredited continuing education at no charge to participants, with a meal provided. Additional funds were needed to purchase prop supplies for OSCE stations and food for sequestered students over the two-day summative OSCE. These additional costs were approved in the year prior to the start of the course, resulting in an increased skills development budget.
Other institutions wishing to implement a similar capstone course should complete a thorough needs assessment, including identification of content and skills to be included, faculty resources to coordinate and teach in the course, faculty training needs (e.g. OSCE development), facilities resources (e.g. space for OSCEs or other simulation-based activities), and financial resources. Strong administration support is essential, as a course of this scope may require increased financial resources relative to most courses, design input from multiple departments, and devoted faculty time from a significant number of faculty. While input from a variety of stakeholders is necessary, the course has benefitted from clear leadership from the course coordinators in terms of organisation and enforcing common expectations across multiple activities.

One critical element in designing a course to assess APPE-preparedness is to establish how students will demonstrate APPE-readiness. For example, the process of OSCE development for this course, in particular setting the summative OSCE cut score, required faculty to be deliberate and to define the minimum level of competence to be demonstrated prior to beginning APPE. Additionally, the letter-graded portions of the course required students to demonstrate additional essential knowledge and skills, including drug information, medical literature evaluation, prescription checking, and calculations. While students were not required to pass each graded assignment individually, students were required to perform globally at a level determined by the faculty to be acceptable to demonstrate APPE-readiness.

Early planning is also crucial in the development of this type of course. For example, given the number of faculty participating in the course, confirmations of availability occurred six months prior to the start of the course to ensure that activity sequencing would flow in a logical order.

Ideally, the structure of the course should be designed to meet the identified needs of the programme. One significant challenge to capstone course design is the perceived need to include all prior content or a significant number of assessments, which may not be possible due to constraints in time or faculty and financial resources. When contact hours, meeting times, or assigned meeting space cannot be adjusted to accommodate the "ideal" course or the need for faculty and financial resources are identified, course designers should seek input from broad faculty groups to determine the essential content and assessments to include, as well as with administrators to address resource needs. 
A unique capstone course that incorporates opportunities for review of essential material, as well as assessment of APPE readiness, was successfully developed and implemented for third-year pharmacy students. The strengths of this course include the use of multiple activities to assess student knowledge and skills and the use of multiple assessment methods, in particular using OSCEs as a formative tool. The scope of the course allowed for broad review but required a significant amount of preparation and participation from faculty. The use of formative OSCEs as a learning method was well-received by students, and student course evaluations suggest that the majority of course elements were perceived to be valuable. Additional opportunities for study related to this course include predictors of student performance in the course, the impact of this course on APPE and NAPLEX performance, as well as stress or anxiety that student experience during the course and while completing OSCEs. Student performance in the course was used to guide changes in future offerings of this course and other courses within the didactic curriculum.

\section{References:}

Abu Bdair, I. A., Abuzaineh, H. F. \& Burqan, M. R. (2019). Advantages and disadvantages of the objective structured clinical examination (OSCE) in nursing education: a literature review. International Journal of Trend in Scientific Research and Development, 3(2), 270. https://doi.org/10.31142/ijtsrd20269

Austin, Z., O’Byrne, C., Pugsley, J., Quero Munoz, L., \& Munoz, L. Q. (2003). Development and validation processes for an objective structured clinical examination (OSCE) for entry-topractice certification in pharmacy: The Canadian experience. American Journal of Pharmaceutical Education, 67(3), 76. https://doi.org/10.5688/aj670376

Beatty, S. J., Kelley, K. A., Ha, J., \& Matsunami, M. (2014). Measuring preadvanced practice experience outcomes as part of a PharmD capstone experience. American Journal of Pharmaceutical Education, 78(8), 152. https://doi.org/10.5688/ajpe788152

Bonsell, R. (2018). NAPLEX Practice Question Workbook. Lexington, KY: Coventry House Publishing

Brazeau, C., Boyd, L., \& Crosson, J. (2002). Changing an existing OSCE to a teaching tool: the making of a teaching OSCE. Academic Medicine, 77(9), 932. https://doi.org/10.1097/00001888200209000-00036

Brooks, A., Sanoski, C., Hajjar, E., \& Overholser, B. (2018). NAPLEX Prep 2018. New York, NY: Kaplan Publishing

Carnegie Mellon University Eberly Center: Teaching Excellence and Educational Innovation. (n.d.). What is the difference between formative and summative assessment?

Cauthon, K. A. B., Attridge, R. L., Urteaga, E. M., Witte, A. P., Cauthon KAB, Attridge RL, ... Witte AP. (2017). An objective structured clinical examination to assess pharmacy resident performance. Innovations in Pharmacy, 8(2), 5. https://doi.org/10.24926/iip.v8i2.510

Conway, J. M., \& Ahmed, G. F. (2012). A pharmacotherapy capstone course to advance pharmacy students' clinical documentation skills. American Journal of Pharmaceutical Education, 76(7), 134. https://doi.org/10.5688/ajpe767134

Farahat, E., Javaherian-Dysinger, H., Rice, G., Schneider, L., Daher, N., \& Heine, N. (2016). Exploring students' perceptions of the educational value of formative Objective Structured Clinical Examination (OSCE) in a nutrition program. Journal of Allied Health, 45(1), 20-26

Gortney, J., Rudolph, M. J., Augustine, J. M., Sease, J. M., Bray, B., Pavuluri, N., \& Wong, F. (2019). National trends in the adoption of pharmacy curriculum outcomes assessment for student assessment and remediation. American Journal of Pharmaceutical Education, 83(6). https://doi.org/10.5688/ajpe83066796

Gupta, P., Dewan, P., Singh, T. (2010). Objective Structured Clinical Examination (OSCE) Revisited. Indian Pediatrics, 47, 911

Hastings, J. K., Flowers, S. K., Pace, A. C., \& Spadaro, D. (2010). An objective standardized clinical examination (OSCE) in an advanced nonprescription medicines course. American Journal of Pharmaceutical Education, 74(6), 98. https://doi.org/10.5688/aj740698

Hirsch, A. C., \& Parihar, H. S. (2014). A capstone course with a comprehensive and integrated review of the pharmacy curriculum and student assessment as a preparation for advanced pharmacy practice experiences. American Journal of Pharmaceutical Education, 78(10), 192. https://doi.org/10.5688/ajpe7810192

Hobson, E. H., Johnston, P. E., \& Spinelli, A. J. (2015). Staging a reflective capstone course to transition PharmD graduates to professional life. American Journal of Pharmaceutical Education, 79(1), 14. https://doi.org/10.5688/ajpe79114

Lee, J. K., Cooley, J. H., Tanner, N. E., Hanauer, C. N., Schiefer, D. M., \& Herrier, R. N. (2014). Development of a pharmacy capstone course from focus groups to advanced patient care. American Journal of Pharmaceutical Education, 78(8), 156. https://doi.org/10.5688/ajpe788156

Salinitri, F. D., O'Connell, M. B., Garwood, C. L., Lehr, V. T., \& Abdallah, K. (2012). An Objective Structured Clinical Examination to Assess Problem-Based Learning. American Journal of Pharmaceutical Education, 76(3), 1-44. https://doi.org/10.5688/ajpe76344

Saseen, J. J., Linnebur, S. A., Borgelt, L. M., Trujillo, J. M., Fish, D. N., \& Mueller, S. W. (2017). A pharmacotherapy capstone course to target student learning and programmatic curricular assessment. American Journal of Pharmaceutical Education, 81(3), $45 . \quad$ Retrieved from https://doi.org/10.5688/ajpe81345

Schwinghammer, T. L., Vice, A. J. C., Boyce, E. G., Bradley, B., Christensen, A., Dunnenberger, H. M., ... Wargo, K. A. (2016). ACCP Toolkit. Pharmacotherapy, 36(11), e189-e194. https://doi.org/10.1002/phar.1846

Shirwaikar, A. (2015). Objective structured clinical examination (OSCE) in pharmacy education - a trend. Pharmacy Practice, 13(4), 627. https://doi.org/10.18549/PharmPract.2015.04.627

Sloan, D., Donnelly, M., Schwartz, R., \& Strodel, W. (1995). The Objective Structured Clinical Examination - the new gold 
standard for evaluating. Annals of Surgery, 222(6), 735-742. https://doi.org/10.1097/00000658-199512000-00007

Sturpe, D. A. (2010). Objective Structured Clinical Examinations in Doctor of Pharmacy programs in the United States. American Journal of Pharmaceutical Education, 74(8), 148. https://doi.org/10.5688/aj7408148

Urteaga, E. M., Attridge, R. L., Tovar, J. M., \& Witte, A. P. (2015). Evaluation of clinical and communication skills of pharmacy students and pharmacists with an objective structured clinical examination. American Journal of Pharmaceutical Education, 79(8), 122. https://doi.org/10.5688/ajpe798122 